\title{
Position-dependent mass quantum systems and ADM formalism
}

\author{
Davood Momeni ${ }^{\star}$ \\ Department of Physics, College of Science, Sultan Qaboos University, \\ P.O. Box 36, Al-Khodh 123, Muscat, Sultanate of Oman \\ $\star$ davood@squ.edu.om
}

4th International Conference on Holography,

String Theory and Discrete Approach

Hanoi, Vietnam, 2020

doi:10.21468/SciPostPhysProc.4

\begin{abstract}
The classical Einstein-Hilbert (EH) action for general relativity (GR) is shown to be formally analogous to the classical system with position-dependent mass (PDM) models. The analogy is developed and used to build the covariant classical Hamiltonian as well as defining an alternative phase portrait for GR. The set of associated Hamilton's equations in the phase space is presented as a first-order system dual to the Einstein field equations. Following the principles of quantum mechanics, I build a canonical theory for the classical general. A fully consistent quantum Hamiltonian for GR is constructed based on adopting a high dimensional phase space. It is observed that the functional wave equation is timeless. As a direct application, I present an alternative wave equation for quantum cosmology. In comparison to the standard Arnowitt-Deser-Misner(ADM) decomposition and quantum gravity proposals, I extended my analysis beyond the covariant regime when the metric is decomposed into the $3+1$ dimensional ADM decomposition. I showed that an equal dimensional phase space can be obtained if one applies ADM decomposed metric.
\end{abstract}

(c) (1) Copyright D. Momeni.

This work is licensed under the Creative Commons

Attribution 4.0 International License.

Published by the SciPost Foundation.
Received 03-08-2020

Accepted 08-01-2021

Published 13-08-2021

doi:10.21468/SciPostPhysProc.4.009

\section{Introduction}

Classical mechanics is Galilean invariant, i.e, time parameter $t$ and position coordinate $q(t)$ are explicitly functions of each other. Since quantum mechanics is Galilean invariant there is no simple way to build a locally Lorentz invariant theory with single particle interpretation (the possible version known as Klein-Gordon has field theoretic realization). In GR, we have difficulty to interpret time as we did in classical mechanics. Furthermore GR is locally Lorentz invariant. The simple reason is that in the context of GR (or any other classical gauge theory for gravity ),time $t$ is just a coordinate and is no longer considered as a parameter (in nonrelativistic mechanics and QM the time $t$ is an evolution parameter). As a result the analogue 
to coordinate (in the variational process), the Riemannian metric $g_{a b}\left(x^{c}\right), a, b=0 \ldots 3$ is a function of the all coordinates $x^{c}=\left(t, x^{A}\right), A=1, . .3$. That makes quantum gravity difficult to construct. A way to address quantum gravity is string theory [1] and the other well studied candidate is loop quantum gravity [2]. There is also semi quantum gravity, when we keep the background classical and let fields simply propagate on it. With both of these nice ideas, still we have the problem of "disappearance of time" [3]. If we adopt any of these proposals, it seems that time problem remains unsolved both in the quantum gravity and cosmology [4]. Time problem has a rather long history [5]. There is a simple way to address quantum gravity without time considered in canonical quantization in metric variables [6]. With all of the above historical backgrounds and many others, we are still looking for a fully covariant canonical quantum theory for gravity which make sense same as we know for usual classical mechanics. It is necessary to find an appropriate representation for Lagrangian of the gravity (here GR as the best tested one ).

With a suitable covariant definition of the conjugate momentum we define a Hamiltonian. Furthermore we need to adopt a well defined phase space. In that phase space one can build Poisson brackets easily, and then by replacing the classical bracket with the Dirac bracket, we can find a suitable fully consistent Hamiltonian for quantum GR. Later, one can build an associated (functional) Hilbert space and develop all the concepts of ordinary quantum mechanics systematically. This is a plan to find a successful quantum theory for gravity or as it is known, quantum gravity. During studying non standard classical dynamical systems I found a class of Lagrangian models with second order time derivative of the position $\ddot{q}(t)$ (configuration coordinate $q(t))$. It is easy to show that a wide class of such models reduce to the position dependence mass (PDM) models as it was investigated in literature [7-9]. It is obviously interesting to show that whether GR reduces to such models. This is what I investigate in this letter. I show that the classical Einstein-Hilbert (EH) Lagrangian reduces to the position-dependent -mass (PDM) model up to a boundary term. Then I adopted the standard quantization scheme for a PDM system and I suggested a fully covariant quantum Hamiltonian for GR. The functional wave equation for the metric proposed naturally and then it was developed for quantum cosmology.

My observations initiated when I studied GR as a classical gauge theory [10]. As everyone knew, GR has a wider class of symmetries provided by the equivalence principle. It respects gauge transformations (any type of arbitrary change in the coordinates, from one frame to the other $x^{a} \rightarrow \tilde{x}^{a}$ ) [10]. Consequently GR is considered just a classical gauge theory for gravity. There is also a trivial hidden analogue between GR and classical mechanics (see table I). In GR as a classical dynamical system (but with second order derivative of the position ), if we make an analogous Riemannian metric $g_{a b}$ with the coordinate $q(t)$ and if we use the spacetime derivatives of the metric $\partial_{c} g_{a b}$ (which is proportional to the Christoffel symbols $\Gamma_{d a b}$ ), instead of the velocity, i.e, the time variation of the coordinate $\dot{q}$, and by adopting a symmetric connection, we can rewrite EH Lagrangian in terms of the metric, first and second derivatives of it. It looks like a classical system in the form of $L(q, \dot{q}, \ddot{q})$ (see TABLE I). In this formal analogy, the classical acceleration term in the classical models under study now $\ddot{q}$ is now replaced with the second derivative for metric i.e, $\partial_{e} \Gamma_{d a b}$. Integration part by part from this suitable representation of the EH Lagrangian reduces it to a PDM system where we will need to define a super mass tensor as a function of the metric instead of the common variable mass function $m(q(t))$ in classical mechanic.

In this letter, I focus on the classical EH action for GR as an analogy to the model investigated in the former above. The notation for Einstein-Hilbert action is

$$
S_{E H}=\int d^{4} x \mathcal{L}_{G R} .
$$

We don't consider matter fields action $S_{\text {matt }}$, although the matter fields are playing crucial 
Table 1: Analogy between classical mechanics and GR

\begin{tabular}{ccccc}
\hline \hline Model & Position & first derivative & second derivative & mass \\
\hline Classical PDM & $q(t)$ & $\dot{q}(t)$ & $\ddot{q}(t)$ & scalar $m(q)$ \\
GR & $g_{a b}$ & $\partial_{d} g_{a b}$ & $\partial_{e} \partial_{d} g_{a b}$ & super mass tensor $M^{a b l d e h}$ \\
& & & given in eq.(9) \\
\hline
\end{tabular}

roles as source for non vacuum classical solution in GR. There is a reason on why we focused on the vacuum GR: If one quantizes the matter sector, the same quantization technique can't be applied to the geometrical part. The resulting theory will be considered as a semi classical qunatum gravity, i.e, it defines a theory where the quantized matter fields propagate on a fully classical background. There is no quantization in any form imposed on the geometry of the spacetime. For example we don't consider non commutative of the spacetime structures or etc. The resulting theory is fully consistent and works as a semi classical regime as it proposed and developed for example in Ref. [11]. Furthermore, the matter Lagrangian is considered non-minimally coupled to the kinetic sector of the theory,we believe the canonical approach used in this paper can't easily explored for theories with matter Lagrangian with non-minimal coupling between geometry and matter fields because they will violate equivalent principle as well as they can't easily be interpreted as unforced position dependence qunatum mechanical system. By the above reasons we only construct a canonical quantization for GR as an empty space theory with non trivial ( at least one) classical geometry. The technique can be applied if one consider locally distributions of the matter field with a local and singular matter energy momentum tensor $T_{\mu \nu} \propto \delta\left(\vec{x}-\vec{x}^{\prime}\right)$ at singularity point $x=x^{\prime}$. For example when the geometry is static, time independent and spherically symmetric with Schwarzschild vacuum solution. The key idea is to realize GR as a classical dynamical system with second order derivative. The Einstein field equations are derived as a standard variational problem subjected to a set of appropriate boundary conditions. Since the idea of GR is to find the best geometry for a given source of matter fields, it is formally equivalent to the classical mechanics. By combining all these similarities I end up to an equivalent representation of $\mathrm{EH}$ action as a classical Lagrangian in the form :

$$
\mathcal{L}_{G R}=L\left(|g|, \partial_{a}|g|, g_{b c}, g^{b c}, \Gamma_{b c}^{a}, \partial_{d} \Gamma_{b c}^{a}\right) .
$$

Here $|g| \equiv \operatorname{det}\left(g_{\mu \nu}\right)$ is determinant of the metric tensor, and $\partial_{\beta}$ is coordinate derivative. We can write the above Lagrangian formally in a more compact form as

$$
\mathcal{L}_{G R}=\mathcal{L}_{G R}(|g|, \partial|g|, g, \bar{g}, \partial g, \partial \bar{g}) .
$$

Here we abbreviated by $g \equiv g_{a b}, \bar{g} \equiv g^{a b},|g| \equiv \operatorname{det}\left(g_{a b}\right)=\frac{1}{\operatorname{det}(\bar{g})}, \frac{1}{2} \bar{g} \cdot \partial g \equiv \Gamma_{b c}^{a}$, $\partial g=\Gamma_{b l a} \equiv g_{b l, a}+g_{l a, b}-g_{b a, l}$. We adopt metricity condition $\nabla_{a} g^{b c}=|g|^{-1 / 2} \partial_{a}\left(|g|^{1 / 2} g^{b c}\right) \equiv|g|^{-1 / 2} \partial\left(|g|^{1 / 2} \bar{g}\right)=0, \partial \cdot \bar{g}=-\bar{g} \cdot \partial g \cdot \bar{g}$. The plan of this letter is as following: In Sec. (2) I showed that GR Lagrangian reduces to a PDM fully classical system with a super mass tensor of rank six. In Sec. (3) I construct a consistent super phase space as well as a set of Poisson brackets. As an attempt to break the complexity of the field equations, I show that gravitational field equations reduced to a set of first order Hamilton's equations. In Sec. (4) I define quantum Hamiltonian simply by replacing the classical brackets with Dirac brackets. The functional wave equation will be proposed and by solving it, we can obtain generic wave function for a fully canonical quantized Riemannian metric. As a concrete example, in Sec. (5) I solve functional wave equation for a cosmological background. Some asymptotic solutions are presented. The last section is devoted to summarize results. 


\section{Super Mass Tensor for GR as PDM classical system}

We adopt the conversion of indices as Ref. [12]. The EH action for GR In units $16 \pi G \equiv 1$ is

$$
S_{E H}=\int_{\mathcal{M}} d^{4} x \sqrt{g} R \equiv \int_{\mathcal{M}} d^{4} x \mathcal{L}_{G R} .
$$

The Ricci scalar $R$ is composed of the metric and its first and second derivatives. The first aim to express the integrand (Lagrange density $\mathcal{L}_{G R}$ ) is as the form from which PDM kinetic term is obvious. We note that the Lagrangian density eq. (5) is a purely kinetic form, with a PDM effective mass. This adequate representation can be obtained from the definition of $\Gamma_{\text {bla }}$, this will be clear if we rewrite the Lagrangian in following equivalent form(note that the Lagrangian enjoys an exchange of indices symmetry $a \rightarrow d$ in the first two terms), in action presented in eq.(3) one can eliminate the second derivative term $\partial_{d e} g_{a b}$ simply by integrating by part and using the metricity condition $\nabla_{a} g^{b c}=0$, by taking into the account all the above requirements a possible equivalent form for Lagrangian of the GR is given by:

$$
\begin{aligned}
\mathcal{L}_{G R}= & \frac{1}{2} \sqrt{|g|}\left(g^{a l} g^{b e} \Gamma_{b l a} \partial^{h} g_{e h}+g^{b e} g^{d h} \Gamma_{b l d} \partial^{l} g_{e h}\right. \\
& \left.+\frac{1}{2} g^{a l} g^{b d} g^{t e} \Gamma_{t l d} \Gamma_{b e a}-\frac{1}{2} g^{a l} g^{b d} g^{t e} \Gamma_{t l a} \Gamma_{b e d}\right),
\end{aligned}
$$

and $S_{E H}=\int d^{4} x \mathcal{L}_{G R}+B . T$ here by B.T we mean boundary term defined as

$$
B . T=\left.\int_{\partial \mathcal{M}} \sqrt{\left|h_{A B}\right|} h^{B D} h^{A L} \Gamma_{B L A}\right|_{x^{D}=\text { constant }}+\left.\int_{\partial \mathcal{M}} \sqrt{\left|h_{A B}\right|} h^{B D} h^{A L} \Gamma_{B L D}\right|_{x^{A}=\text { constant }} .
$$

We can re express the above GR Lagrangian in our convenient notations as

$$
\mathcal{L}_{G R}=\frac{1}{2} \sqrt{|g|}\left(\bar{g} \cdot \partial g \cdot \bar{g} \cdot \bar{\partial} g+\bar{g} \cdot \bar{g} \cdot \partial g \cdot \bar{\partial} g+\frac{1}{2} \bar{g} \cdot \bar{g} \cdot \bar{g} \cdot \partial g \cdot \partial g-\frac{1}{2} \bar{g} \cdot \partial g \cdot \bar{g} \cdot \bar{g} \cdot \partial g\right) .
$$

Note that by "." we mean tensor product(we adopt Einstein summation rule). From the above representation we can realize $\{g, \bar{g}\}$ as two fields, in analogy to the Dirac Lagrangian where the fermionic pairs $\psi, \bar{\psi}$ appeared. The difference here is due to the fact that the pair of objects $g, \bar{g}$ depend on each other as we know $g \cdot \bar{g}=\delta$, the Kronecker delta, however in the Dirac Lagrangian the norm $\bar{\psi} \psi \neq I$. In our program we wont use this duality and we will focus on the coordinates representation of the GR Lagrangian, i.e, eq.(5). If we substitute the definition of Gamma terms and combine the theory, we obtain the final form for the Lagrangian as a PDM system for coordinate $g_{a b}$ (or as a tensor version for k-essence [13]):

$$
\mathcal{L}_{G R}=\frac{1}{2} \sqrt{|g|} M^{\text {abldeh }} \partial_{a} g_{b l} \partial_{d} g_{e h}
$$

Here $M^{\text {abldeh }}=|g|^{-1 / 2} \frac{\partial^{2} \mathcal{L}_{G R}}{\partial\left(\partial_{a} g_{b l}\right) \partial\left(\partial_{d} g_{e h}\right)}$ is defined as super mass tensor. This super mass tensor previously introduced in $[14,15]$. In both the Refs. $[14,15]$, the authors the reduction of the gravitational action in the favor of the horizon thermodynamics and mainly to explain the features of the emergent gravity paradigm. An alternative form for (8) is $\mathcal{L}_{G R}=\frac{\sqrt{|g|}}{2} \bar{M} \partial g \partial g$. It is equivalent to the classical Lagrangian of PDM systems $L=\frac{1}{2} M(q) \dot{q}^{2}$ for one dimensional, position dependent mechanical system. As we expected in GR, the mass term transformed to 
a higher order (here rank six) tensor. The explicit form for the super mass tensor is expressed as following:

$$
\begin{aligned}
& |g|^{1 / 2} \bar{M}=|g|^{1 / 2} M^{a_{1} b_{1} l_{1} d_{1} e_{1} h_{1}}=\frac{1}{4} g^{a l} g^{b d} g^{t e} \times \\
& \left(\delta_{a}^{a_{1}} \delta_{b}^{b_{1}} \delta_{e}^{l_{1}}+\delta_{a}^{b_{1}} \delta_{b}^{a_{1}} \delta_{e}^{l_{1}}-\delta_{a}^{l_{1}} \delta_{b}^{b_{1}} \delta_{e}^{a_{1}}\right)\left(\delta_{d}^{d_{1}} \delta_{l}^{h_{1}} \delta_{t}^{e_{1}}-\delta_{d}^{h_{1}} \delta_{l}^{d_{1}} \delta_{t}^{e_{1}}+\delta_{d}^{e_{1}} \delta_{l}^{h_{1}} \delta_{t}^{d_{1}}\right) \\
& -\frac{1}{4} g^{a l} g^{b d} g^{t e}\left(\delta_{b}^{a_{1}} \delta_{d}^{b_{1}} \delta_{e}^{l_{1}}+\delta_{b}^{b_{1}} \delta_{d}^{a_{1}} \delta_{e}^{l_{1}}-\delta_{b}^{b_{1}} \delta_{d}^{l_{1}} \delta_{e}^{a_{1}}\right)\left(\delta_{a}^{d_{1}} \delta_{l}^{h_{1}} \delta_{t}^{e_{1}}-\delta_{a}^{h_{1}} \delta_{l}^{d_{1}} \delta_{t}^{e_{1}}+\delta_{a}^{e_{1}} \delta_{l}^{h_{1}} \delta_{t}^{d_{1}}\right) \\
& \left.+\frac{1}{4} g^{a l} g^{b d} g^{t e}\left(\delta_{a}^{d_{1}} \delta_{b}^{e_{1}} \delta_{e}^{h_{1}}+\delta_{a}^{e_{1}} \delta_{b}^{d_{1}} \delta_{e}^{h_{1}}-\delta_{a}^{h_{1}} \delta_{b}^{e_{1}} \delta_{e}^{d_{1}}\right)\left(\delta_{d}^{a_{1}} \delta_{l}^{l_{1}} \delta_{t}^{b_{1}}-\delta_{d}^{l_{1}} \delta_{l}^{a_{1}} \delta_{t}^{b_{1}}+\delta_{d}^{b_{1}} \delta_{l}^{l_{1}} \delta_{t}^{a_{1}}\right)\right) \\
& \left.-\frac{1}{4} g^{a l} g^{b d} g^{t e}\left(\delta_{b}^{d_{1}} \delta_{d}^{e_{1}} \delta_{e}^{h_{1}}+\delta_{b}^{e_{1}} \delta_{d}^{d_{1}} \delta_{e}^{h_{1}}-\delta_{b}^{e_{1}} \delta_{d}^{h_{1}} \delta_{e}^{d_{1}}\right)\left(\delta_{a}^{a_{1}} \delta_{l}^{l_{1}} \delta_{t}^{b_{1}}-\delta_{a}^{l_{1}} \delta_{l}^{a_{1}} \delta_{t}^{b_{1}}+\delta_{a}^{b_{1}} \delta_{l}^{l_{1}} \delta_{t}^{a_{1}}\right)\right) \\
& +g^{a l} \delta_{a}^{d_{1}} g^{b e} g^{d h} \delta_{e}^{e_{1}} \delta_{h}^{h_{1}}\left(\delta_{b}^{b_{1}} \delta_{d}^{a_{1}} \delta_{l}^{l_{1}}+\delta_{b}^{a_{1}} \delta_{d}^{l_{1}} \delta_{l}^{b_{1}}-\delta_{b}^{b_{1}} \delta_{d}^{l_{1}} \delta_{l}^{a_{1}}\right) \\
& +g^{a l} g^{b e} g^{d h} \delta_{d}^{d_{1}} \delta_{e}^{e_{1}} \delta_{h}^{h_{1}}\left(\delta_{a}^{a_{1}} \delta_{b}^{b_{1}} \delta_{l}^{l_{1}}+\delta_{a}^{l_{1}} \delta_{b}^{a_{1}} \delta_{l}^{b_{1}}-\delta_{a}^{l_{1}} \delta_{b}^{b_{1}} \delta_{l}^{a_{1}}\right) \\
& +g^{a l} \delta_{a}^{a_{1}} g^{b e} g^{d h} \delta_{e}^{b_{1}} \delta_{h}^{l_{1}}\left(\delta_{b}^{e_{1}} \delta_{d}^{d_{1}} \delta_{l}^{h_{1}}+\delta_{b}^{d_{1}} \delta_{d}^{h_{1}} \delta_{l}^{e_{1}}-\delta_{b}^{e_{1}} \delta_{d}^{h_{1}} \delta_{l}^{d_{1}}\right) \\
& +g^{a l} g^{b e} g^{d h} \delta_{d}^{a_{1}} \delta_{e}^{b_{1}} \delta_{h}^{l_{1}}\left(\delta_{a}^{d_{1}} \delta_{b}^{e_{1}} \delta_{l}^{h_{1}}+\delta_{a}^{h_{1}} \delta_{b}^{d_{1}} \delta_{l}^{e_{1}}-\delta_{a}^{h_{1}} \delta_{b}^{e_{1}} \delta_{l}^{d_{1}}\right) \text {. }
\end{aligned}
$$

Having the Lagrangian of GR given in eq. (8), one can define a canonical pair of position conjugate momentum $(g, \bar{p})$ and construct a phase space. This is what we are going to do in next section. Before to have more process, we would like to motivate of our study. If one can reduce the GR to a Hamiltonian form, the reduced form can be used for example in the numerical relativity and canonical quantization. The Hamiltonian formulation is a preferred approach to study dynamics of the classical systems as well as an attempt to quantization of the models. As we know the Hamiltonian formulation of GR requires a $3+1$ decomposition of the space and time coordinates. We refer the readers to the mini review on the subject presented by [16] for more discussions and seeing of how the Hamiltonian formalism works in classical backgrounds.

\section{Super phase space}

The phase space description of the classical model presented in eq.(8) is very straightforwardly done, by defining the super conjugate momentum tensor is

$$
p^{r s t}=\frac{\partial \mathcal{L}_{G R}}{\partial\left(\partial_{r} g_{s t}\right)}=\frac{\sqrt{g}}{2}\left(M^{r s t d e h} \partial_{d} g_{e h}+M^{a b l r s t} \partial_{a} g_{b l}\right) .
$$

Note that the mass tensor $M^{r s t d e h} \partial_{d} g_{e h}=M^{a b l r s t} \partial_{a} g_{b l}$. A possible classical Hamiltonian will be

$$
\mathcal{H}_{G R}=\frac{1}{2 \sqrt{|g|}} M^{a b l d e h} M_{r s t a b l} p^{r s t} M_{u v w d e h} p^{u v w} .
$$

A possible Poisson's bracket $\{F, G\}_{P . B}$ adopted to this system is:

$$
\left\{F\left(g_{m n}, p^{s t u}\right), G\left(g_{m n}, p^{s t u}\right)\right\}_{P . B}=\sum\left(\frac{\partial F}{\partial g_{a b}} \frac{\partial G}{\partial p^{r s t}}-\frac{\partial F}{\partial p^{r s t}} \frac{\partial G}{\partial g_{a b}}\right),
$$

or in our notation it simplifies to the following expression

$$
\{F(g, \bar{p}), G(g, \bar{p})\}_{P . B}=\sum\left(\frac{\partial F}{\partial g} \frac{\partial G}{\partial \bar{p}}-\frac{\partial F}{\partial \bar{p}} \frac{\partial G}{\partial g}\right)
$$


and specifically for our super phase coordinates $\left(g_{a b}, p^{r s t}\right)$, I postulate that

$$
\left\{g_{a b}, p^{r s t}\right\}_{P . B}=c^{r} \delta_{a b}^{s t} .
$$

Here $\delta_{a b}^{s t}$ is the generalized Kronecker defined as [17]

$$
\delta_{a b}^{s t}=2 ! \delta_{[a}^{s} \delta_{b]}^{t} .
$$

In the above Poisson's bracket, with structure constants $c^{r}$ provide a classical minimal volume for super phase space (zero for Poisson's bracket same objects ). We have now full algebraic structures in the super phase space and canonical Hamiltonian. As a standard procedure, we can write down Hamilton's equations as first order reductions of the Euler-Lagrange equations derived from the Lagrangian given in eq. (8) (Einstein field equations). This is one of the main results of this letter and I will address it in the next short section.

\subsection{Reduction of the Einstein Field Equations (EFE) to Hamilton's equation via covariant Hamiltonian}

The set of Hamilton's equations derived from the Hamiltonian (11), are defined automatically using the Poisson's bracket are given as following:

$$
\begin{aligned}
& \partial_{a} g_{b l}=\left\{g_{b l}, \mathcal{H}_{G R}\right\}_{P . B}=\frac{\partial \mathcal{H}_{G R}}{\partial p_{a b l}}, \\
& \partial_{a} p^{a b l}=\left\{p^{a b l}, \mathcal{H}_{G R}\right\}_{P . B}=-\frac{\partial \mathcal{H}_{G R}}{\partial g_{b l}} .
\end{aligned}
$$

We explicitly can write this pair of Hamilton's equations given as follows:

$$
\begin{aligned}
& \quad \frac{2}{\sqrt{g}} \partial_{a} g_{b l}=M^{a^{\prime} b^{\prime} l^{\prime} d e h} M_{r s t a^{\prime} b^{\prime} l^{\prime}} M_{u v w d e h} \times\left(\delta_{a}^{u} \delta_{b}^{v} \delta_{l}^{w} p^{r s t}+\delta_{a}^{r} \delta_{b}^{s} \delta_{l}^{t} p^{u v w}\right) \\
& -\frac{2}{\sqrt{g}} \partial_{a} p^{a b l}=M^{a^{\prime} b^{\prime} l^{\prime} d e h} p^{r s t} M_{r s t a^{\prime} b^{\prime} l^{\prime}} p^{u v w} \frac{\partial M_{u v w d e h}}{\partial g_{b l}}+M^{a^{\prime} b^{\prime} l^{\prime} d e h} p^{r s t} M_{u v w d e h} \frac{\partial M_{r s t a^{\prime} b^{\prime} l^{\prime}}}{\partial g_{b l}} \\
& +\frac{\partial M_{a^{\prime} b^{\prime} l^{\prime} d e h}}{\partial g_{b l}} p^{r s t} M_{r s t a^{\prime} b^{\prime} l^{\prime}} p^{u v w} M_{u v w d e h}+M^{a^{\prime} b^{\prime} l^{\prime} d e h} \frac{\partial p^{r s t}}{\partial g_{b l}} M_{r s t a^{\prime} b^{\prime} l^{\prime}} p^{u v w} M_{u v w d e h} \\
& +M^{a^{\prime} b^{\prime} l^{\prime} d e h} p^{r s t} M_{r s t a^{\prime} b^{\prime} l^{\prime}} \frac{\partial p^{u v w}}{\partial g_{b l}} M_{u v w d e h} .
\end{aligned}
$$

This set of first order partial differential equations are considered the first phase space alternative to the original gravitational field equations. When we succeed to write a covariance Hamiltonian, the Hamilton's equations are first order version of the Einstein field equations. Reduction of the Einstein field equation to a system of the first order Hamiltonian equations were investigated in several former works. For example in the classical Ref. [18], a 3+1 decomposition technique introduced to explore a possible Hamiltonian formulation of the GR. The general ideas about how to write Hamilton constraints discussed by Dirac in [19]. Following Dirac's idea, later the authors in Ref. [20] proved that for GR, a set of the canonical variables existed only for a given restricted submanifold. This submanifold is specified by putting all the canonical variables equal to zero. In the important work by DeWitt [6], the author presented a conventional canonical formulation of GR. The classical field theory perspective introduced in Ref. [21] along the standard other gauge theories. In comparison to the CMB anisotropies in the standard cosmology, the Hamiltonian formalism used to compute the anisotropies [22]. They showed that how such Hamiltonian formalism helps us to calculate the model predictions in linear theory for any standard classical cosmological background. The Hamiltonian 
formalism extensively introduced and applied to calculate cosmological perturbations in cosmological backgrounds in Ref [23]. The set of equations given in $(16,17)$ are defined when a first order Hamiltonian version of the field equations for a generic Lorentzian metric. I believe that one can integrate this system as a general non autonomous dynamical system for a given set of the appropriate initial values of the metric and super momentum given as a specific initial position $x_{0}^{a}$ (not specific time which is commonly considered as the initial condition in QG literature). A remarkable observation is that the system may possess chaotic behavior and doesn't suffer from Cauchy's problem. We have now the classical Hamiltonian and the set of Poisson brackets. Now we can develop a qunatum version and obtain qunatum Hamiltonian for GR. This will be done in the next section.

\section{Quantization of GR}

In this section, I'm going to define appropriate forms for Dirac brackets simply by defining,

$$
\begin{aligned}
& \hat{\pi}^{r s t} \equiv-i \hbar^{r} \frac{\partial}{\partial \hat{g}_{s t}}, \\
& {\left[\hat{g}_{a b}, \hat{\pi}^{r s t}\right]=i \hbar^{r} \delta_{a b}^{s t} .}
\end{aligned}
$$

Instead of the usual fundamental reduced Planck's constant $\hbar$ we required to define a vector form of the reduced Planck's constant $\hbar^{r}$. The reason is that even the classical phase space spanned by the $\left(g_{a b}, p^{r s t}\right)$ has more degrees of freedom (dof), basically the total dof is $10^{5}\left(=10 \times 10^{4}\right)$ dimensional for a Riemannian manifold. The Dirac constant $\hbar$ is proportional to the minimum volume of the phase space $V_{0}$ defined as

$$
\omega_{0}=\int D\left(g_{a b}, p^{r s t}\right)
$$

where the $D\left(g_{a b}, p^{r s t}\right)$ is a measure for the super phase space and $D\left(g_{a b}, p^{r s t}\right)$ is a covariant volume element. We obviously see that the $\omega_{0}$ is related to the dof of the system, for example if the system has $f$ numbers of dof, then the minimal volume of the phase space is given as $\hbar^{f}$ and here $\hbar \propto\left(\omega_{0}\right)^{1 / f}$, note that in our new formalism $f=10^{5} \gg 1$, as a result the effective $\left\|\hbar^{r}\right\| \ll \hbar$.

A remarkable observation is that the super mass tensor $\bar{M}=M^{\text {abldeh }}$ is a homogeneous (order 6) of the metric tensor. Using the formalism of quantization for PDM systems the canonical quantized Hamiltonian for GR is:

$$
\hat{\mathcal{H}}_{G R}\left(\hat{g}_{a b}, \frac{\partial}{\partial \hat{g}_{s t}}\right)=-\frac{1}{2} f_{r s t u v w}^{1 / 2} \hbar^{r} \frac{\partial}{\partial \hat{g}_{s t}}\left[f_{r s t u v w}^{1 / 2} \hbar^{u} \frac{\partial}{\partial \hat{g}_{v w}}\right] .
$$

Here the auxiliary, scaled super mass tensor $f_{r s t u v w}$ is defined as bellows,

$$
f_{\text {rstuvw }} \equiv|g|^{-1 / 4} M^{\text {abldeh }} M_{\text {rstabl }} M_{\text {uvwdeh }} .
$$

It is adequate to write the quantum Hamiltonian in the following closed form:

$$
\hat{\mathcal{H}}_{G R}\left(\hat{g}_{a b}, \hat{\pi}^{m n p}\right)=\frac{1}{2}\left[|g|^{-1 / 2} \bar{M} M M\right]^{1 / 2} \overline{\hat{\pi}}\left[|g|^{-1 / 2} \bar{M} M M\right]^{1 / 2} \overline{\hat{\pi}},
$$

where $\bar{p}$ is contravariant component of the super momentum $p$, etc. The above quantization of Hamiltonian is covariant since we didn't specify time $t$ from the other spatial coordinates $x^{A}$. The model is considered as a timeless model, i.e, there is no first order time derivative 
in the final wave equation like $\frac{\partial}{\partial t}$, and the associated functional second order wave equation which is fully locally Lorentz invariant as well as general covariant is expressed as:

$$
-\frac{1}{2} f_{r s t u v w}^{1 / 2} \hbar^{r} \frac{\partial}{\partial \hat{g}_{s t}}\left[f_{r s t u v w}^{1 / 2} \hbar^{u} \frac{\partial}{\partial \hat{g}_{v w}} \Psi\left(\hat{g}_{a b}\right)\right]=E \Psi\left(\hat{g}_{a b}\right) .
$$

Note that in our suggested functional wave equation for $\Psi\left(\hat{g}_{a b}\right)$, we end up by the covariant (no first order derivative) of the functional Hilbert space, furthermore all the physical states are static (i.e., no specific time dependency) and consequently we have a covariant full evolution for our functional. We should emphasize here that the theory which we studied in this paper is considered as an attempt to construct quantum mechanics on a classical GR background (see for example independent works in Ref. [24]). There is no simple field-theoretic interpretation for the Hamiltonian which we obtained in this work as well as any other brackets are simply non-quantum field theoretical ones. In this approach, we can't reach the renormalizability as it has been investigated in many other alternative quantum gravity scenarios. We believe that this toy model is a subclass of the timeless models of QG. Building quantum gravity via timeless phase space investigated in the past by some authors mainly recent work [25]. Our approach is completely different and independent from the others. We will study qunatum cosmology as a direct application of our wave equation in the next section.

\section{Quantum cosmology}

In flat, Friedmann-Lemaître-Robertson-Walker (FLRW) model with Lorentzian metric $g_{a b}=\operatorname{diag}\left(1,-a(t) \Sigma_{3}\right)$ where $\Sigma_{3}$ is the unit metric tensor for flat space, in coordinates $x^{a}=(t, x, y, z)$, the non vanishing elements of the super mass tensor defined in eq.(9),

$$
M^{a b l d e h}=-12 a^{-2} \delta^{a 0} \delta^{d 0} \delta^{B L} \delta^{E H},
$$

here $B, L, E, H=1,2,3$ and the auxiliary scaled super mass tensor $f_{\text {rstuvw }}$

$$
f_{r s t u v w}=-\frac{3 a^{1 / 2}}{4} \delta_{u 0} \delta_{r 0} \delta_{V W} \delta_{S T}
$$

The functional wave equation reduces to the hypersurfaces $\sigma_{3}$ coordinates $X^{A}=(x, y, z)$ :

$$
\frac{3 \hbar_{0}^{2} a^{1 / 2}}{8} \frac{\partial^{2} \Psi\left(\hat{g}_{A B}\right)}{\partial \hat{g}_{S S} \partial g_{V V}}=E \Psi\left(\hat{g}_{A B}\right)
$$

and in the coordinates for FLRW metric it reduces simply to the following ordinary differential equation

$$
a \Psi^{\prime \prime}(a)-\Psi^{\prime}(a)-\frac{32 E a^{5 / 2}}{3 h_{0}^{2}} \Psi(a)=0 .
$$

Here prime denotes derivative with respect to the $a$. If we know boundary conditions, one can construct an orthonormal set of eigenfunctions using the Gram Schmidt process. Furthermore the above single value wave equation can be reduce to a standard second order differential equation for wave function $\Psi(a)=\sqrt{a} \phi(a)$,

$$
\phi^{\prime \prime}(a)-\left(\frac{32 E a^{1 / 2}}{3 h_{0}^{2}}+\frac{3}{4 a^{2}}\right) \phi(a)=0 .
$$


It is hard to find an exact solution for the above wave equation but there are exact solutions for asymptotic regimes:

$$
\phi(a) \propto\left\{\begin{array}{ll}
a^{\frac{3}{2}} & \text { if } a \rightarrow 0 \\
\exp \left[\frac{16 \sqrt{2 E}}{5 \sqrt{3} h_{0}} a^{\frac{5}{4}}\right] & \text { if } a \rightarrow \infty
\end{array},\right.
$$

and one can build an exact solution via Poincare's asymptotic technique, i.e, by suggesting

$$
\phi(a)=\zeta(a) a^{\frac{3}{2}} e^{\frac{16 \sqrt{2 E}}{5 \sqrt{3} h_{0}} a^{\frac{5}{4}}}
$$

and $\zeta(a)$ will come as a transcendental (hypergeometric) function

$$
\zeta(a)=\frac{3^{2 / 5} \hbar^{4 / 5} e^{-\frac{2}{15}\left(\frac{8 \sqrt{6 e} e^{5 / 4}}{\hbar}+3\right)}}{64 \sqrt[5]{5} a}\left(C_{1} I_{-\frac{4}{5}}\left(\frac{16 \sqrt{\frac{2 e}{3}} a^{5 / 4}}{5 \hbar}\right)+C_{2} I_{\frac{4}{5}}\left(\frac{16 \sqrt{\frac{2 e}{3}} a^{5 / 4}}{5 \hbar}\right)\right),
$$

here $I_{v}(y)$ is the modified Bessel function of the first kind and the eigenvalue $E$ (positive, negative or zero ) can be discrete as well as continuous (bound states for $E<0$ ). Remarkable is for vanishing energy state, $E=0$, the generic wave function is given by

$$
\Psi(a)=N_{0}+N a^{2}, \quad a \in[0, \infty) .
$$

The above-approximated wave functions obtained for an empty spacetime. In classical cosmology, there are no dynamics for a cosmological background without including matter fields. The resulting FLRW equations simply lead to a static Universe with zero Hubble's parameter $H=0$. It shows that there is no stable equilibrium around this static Universe. The solution is fully rejected by considering cosmological facts. In our quantum mechanical model, we are dealing with wave function instead of the unique classical scale factor or Hubble's parameter. In comparison to the simple Harmonic oscillator in standard QM, the wave function obtained in the above cases give us a non zero scale factor or Hubble's parameter. That is considerable because in ordinary QM, the wave function provides possible probabilistic access to the forbidden regions of the system with inaccessible classical energy regimes. In our case, the following wave functions simply can be understood as we do in QM. In quantum FLRW formalism, the wave function even for ground state as it has been obtained in expression (34) gives non-classical expectation values for different quantities. If for example one compute the $<\hat{\mathcal{H}}_{G R}\left(\hat{g}_{a b}, \hat{\pi}^{m n p}\right)>$, it shows extended amplitudes for the quantum wave function for the Universe. Is also possible to define (formally) uncertainty expressions for $\Delta a, \Delta p_{a}$ and based on the above discussions those exact solution for wave functions are somehow still useful in the absence of a full quantum gravity scenario based on a preferred time foliation.

\section{Note about ADM decomposition formalism and reduced phase space}

Working with an extended phase space with a conjugate momentum with one more index doesn't look very friendly at all, although that is the unique way to define a fully covariant form for the phase space as well as a purely kinetic Lagrangian for GR. If one adopt the ADM decomposition of the metric $g_{a b}$ as follows [26],

$$
d s^{2}=g_{a b} d x^{a} d x^{b}=h_{A B} d x^{A} d x^{B}+2 N_{A} d x^{A} d x^{0}+\left(-N^{2}+h^{A B} N_{A} N_{B}\right)\left(d x^{0}\right)^{2},
$$

here $x^{0}$ is time, $A, B=1,2,3$ refer to the spatial coordinates and $h_{A B}$ is spatial metric. It is easy to show that the set of the first order Hamilton's equations presented in the previous section 
reduces to the $\mathrm{ADM}$ equations, only if one consider $t$ as dynamical time evolution. Basically if we recall the super conjugate momentum

$$
\pi^{r s}=\frac{\partial \mathcal{L}_{G R}}{\partial \dot{g}_{s t}}=\frac{\sqrt{g}}{2}\left(M^{0 s t d e h} \partial_{d} g_{e h}+M^{a b l 0 s t} \partial_{a} g_{b l}\right) .
$$

Builiding the Hamiltonian in a standard format as

$$
\mathcal{H}_{G R}^{A D M}=\frac{1}{2 \sqrt{|g|}} M^{a b l d e h} M_{0 s t a b l} \pi^{s t} M_{0 v w d e h} \pi^{v w} .
$$

Briefly I wanna to mention here that although my formalism is worked with covariant derivative without specifying any coordinate as time (so technically is a timeless technique) if one turns back to the standard metric decomposition in $\mathrm{ADM}$ and use the 0 component of super conjugate momentum, again we can recover ADM Hamiltonian. I emphasis here that my construction was based on purely geometrical quantization of the GR action rather opting a standard time coordinate .

\section{Final remarks}

The canonical covariant quantization which I proposed here is a consistent theory. I started it by basic principles, just by rewriting the GR action in a suitable form the Lagrangian reduced to a purely kinetic theory with position dependence mass term. In this equivalent form of the Lagrangian, gradient of the metric tensor appears as a hypothetical scalar field. With such a simple quadratic Lagrangian, I defined a conjugate momentum corresponding to the metric tensor. The mass term for graviton derived as tensor of rank six . I developed a classical Hamiltonian using the metric and its conjugate momentum. It is remarkable that one can write classical Hamilton's equations for metric and momentum (super phase space coordinates) are analogous to the second order nonlinear Einstein field equations. Later I replaced Poisson's brackets with Moyal(Dirac) and I defined a quantum Hamiltonian for GR. There is no time problem in this formalism because theory is fully covariant from the beginning. As a direct application I investigated qunatum cosmology, i.e and wave function for a homogeneous and isotropic Universe. I showed that wave equation simplifies to a linear second order ordinary differential equation with appropriate asymptotic solutions for very early and late epochs. In my letter I used an integration part by part to reduce GR Lagrangian to a form with first derivatives of the metric. The price is to define two boundary terms on the spatial boundary regions. Those terms vanish in any asymptotic flat(regular) metric. I notice here that even if we didn't remove second derivative terms using integration by part, it was possible to define a second conjugate momentum $r^{a b c d}=\frac{\partial \mathcal{L}_{G R}}{\partial\left(\partial_{a} \partial_{b} g_{c d}\right)}$ corresponding to the second derivative of the metric $\partial_{a} \partial_{b} g_{c d}$. If I impose a Bianchi identity between $g_{a b}, p^{r s t}, r^{a b c d}$, it is possible to fix this new momentum in terms of the other one and the metric. A suitable Legendre transformation from the GR Lagrangian

$$
\mathcal{H}_{G R}=p^{r s t} \partial_{r} g_{s t}+r^{a b c d}\left(\partial_{a} \partial_{b} g_{c d}\right)-\mathcal{L}_{G R},
$$

with the Bianchi identity,

$$
\left\{g_{a b},\left\{p^{c d e}, r^{f g h i}\right\}_{P . B}\right\}_{P . B}+\left\{p^{c d e},\left\{r^{f g h i}, g_{a b}\right\}_{P . B}\right\}_{P . B}+\left\{r^{f g h i},\left\{g_{a b}, p^{c d e}\right\}_{P . B}\right\}_{P . B}=0 .
$$

I obtain a standard Hamiltonian without this new higher order momentum. Consequently the method of finding a Hamiltonian based on the super phase spaces which I defined by $g_{a b}, p^{r s t}$ in Sec. II and the one with one more conjugate momentum i.e, $g_{a b}, p^{r s t}, r^{a b c d}$ leads to the same result. 


\section{Acknowledgments}

This work supported by the Internal Grant (IG/SCI/PHYS/20/07) provided by Sultan Qaboos University. I thank Profs. Lawrence P. Horwitz, John. R. Morris for carefully reading my first draft, very useful comments, corrections and discussions. The tensor manipulations of this done using WXMAXIMA platform. I thank Prof. T. Padmanabhan for remembering his previous relevant works.

\section{References}

[1] E. Silverstein, TASI Lectures on cosmological observables and string theory, New Frontiers in Fields and Strings, World Scientific (2016), doi:10.1142/9789813149441_0009.

[2] C. Rovelli, Loop quantum gravity, Living Rev. Relativ. 11, 5 (2008), doi:10.12942/1rr2008-5.

[3] J. Barbour, The timelessness of quantum gravity: I. The evidence from the classical theory, Class. Quant. Grav. 11, 2853 (1994), doi:10.1088/0264-9381/11/12/005.

[4] D. Braddon-Mitchell and K. Miller, Quantum gravity, timelessness, and the contents of thought, Philos Stud 176, 1807 (2019), doi:10.1007/s11098-018-1097-4.

[5] C. Kiefer, Quantum gravity, Oxford University Press, Int. Ser. Monogr. Phys. 155 (2012).

[6] B. S. DeWitt, Quantum theory of gravity. I. The canonical theory, Phys. Rev. 160, 1113 (1967), doi:10.1103/PhysRev.160.1113.

[7] J. R. Morris, New scenarios for classical and quantum mechanical systems with positiondependent mass, Quantum Stud.: Math. Found. 2, 359 (2015), doi:10.1007/s40509015-0037-7.

[8] Á. Ballesteros, I. Gutiérrez-Sagredo and P. Naranjo, On Hamiltonians with positiondependent mass from Kaluza-Klein compactifications, Phys. Lett. A 381, 701 (2017), doi:10.1016/j.physleta.2016.12.040.

[9] J. F. Cariñena, M. F. Rañada and M. Santander, Quantization of Hamiltonian systems with a position dependent mass: Killing vector fields and Noether momenta approach, J. Phys. A: Math. Theor. 50, 465202 (2017), doi:10.1088/1751-8121/aa8e90.

[10] M. Carmeli, Classical fields: General relativity and gauge theory, John Wiley and Sons (1982).

[11] V. Mukhanov, S. Winitzki, Introduction to quantum effects in gravity, Cambridge University Press, ISBN:9780521868341 (2007).

[12] R. Adler, M. Bazin, M. Schiffer, Introduction to general relativity, McGraw Hill, Tokyo (1975).

[13] C. Armendariz-Picon, V. Mukhanov and P. J. Steinhardt, Essentials of k-essence, Phys. Rev. D 63, 103510 (2001), doi:10.1103/PhysRevD.63.103510.

[14] K. Parattu, B. R. Majhi and T. Padmanabhan, Structure of the gravitational action and its relation with horizon thermodynamics and emergent gravity paradigm, Phys. Rev. D 87, 124011 (2013), doi:10.1103/PhysRevD.87.124011. 
[15] T. Padmanabhan, General relativity from a thermodynamic perspective, Gen. Relativ. Gravit. 46, 1673 (2014), doi:10.1007/s10714-014-1673-7.

[16] E. Bertschinge, Hamiltonian formulation of general relativity, Massachusetts Institute of Technology, http://web.mit.edu/edbert/GR/gr11.pdf.

[17] R. L. Agacy, Generalized Kronecker and permanent deltas, their spinor and tensor equivalents and applications, J. Math. Phys. 40, 2055 (1999), doi:10.1063/1.532851.

[18] Charles W. Misner, K. S. Thorne, J. A. Wheeler and D. I. Kaiser, Gravitation, Princeton University Press (2017).

[19] P. A. M. Dirac, Fixation of coordinates in the Hamiltonian theory of gravitation, Phys. Rev. 114, 924 (1959), doi:10.1103/PhysRev.114.924.

[20] T. Maskawa and H. Nakajima, Singular lagrangian and the Dirac-Faddeev method: Existence theorem of constraints in 'Standard Form', Progr. Theor. Phys. 56, 1295 (1976), doi:10.1143/PTP.56.1295.

[21] S. Weinberg, The quantum theory of fields, Cambridge Univ. Press (1995).

[22] W. Hu, U. Seljak, M. White and M. Zaldarriaga, Complete treatment of CMB anisotropies in a FRW universe, Phys. Rev. D 57, 3290 (1998), doi:10.1103/PhysRevD.57.3290.

[23] E. Bertschinger, Cosmological dynamics, in Cosmology and Large Scale Structure, proc. Les Houches Summer School, Session LX, ed. R. Schaeffer, J. Silk, M. Spiro, and J. ZinnJustin, Elsevier Science, Amsterdam, 273 (1996).

[24] L. P. Horwitz, An elementary canonical classical and quantum dynamics for general relativity, Eur. Phys. J. Plus 134, 313 (2019), doi:10.1140/epjp/i2019-12689-7.

[25] H. Gomes, Quantum gravity in timeless configuration space, Class. Quantum Grav. 34, 235004 (2017), doi:10.1088/1361-6382/aa8cf9.

[26] R. Arnowitt, S. Deser and C. Misner, Dynamical structure and definition of energy in general relativity, Phys. Rev. 116, 1322 (1959), doi:10.1103/PhysRev.116.1322. 